\title{
Multiple positive solutions for a quasilinear system of Schrödinger equations
}

\author{
Giovany M. Figueiredo and Marcelo F. Furtado
}

\begin{abstract}
We consider the quasilinear system

$$
\left\{\begin{array}{l}
-\varepsilon^{p} \operatorname{div}\left(|\nabla u|^{p-2} \nabla u\right)+V(z) u^{p-1}=Q_{u}(u, v)+\gamma H_{u}(u, v) \text { in } \mathbb{R}^{N}, \\
-\varepsilon^{p} \operatorname{div}\left(|\nabla v|^{p-2} \nabla v\right)+W(z) v^{p-1}=Q_{v}(u, v)+\gamma H_{v}(u, v) \text { in } \mathbb{R}^{N}, \\
u, v \in W^{1, p}\left(\mathbb{R}^{N}\right), \quad u(z), v(z)>0 \text { for all } z \in \mathbb{R}^{N},
\end{array}\right.
$$

where $\varepsilon>0,2 \leq p<N, V$ and $W$ are positive continuous potentials, $Q$ is an homogeneous function with subcritical growth, $H(u, v)=|u|^{\alpha}|v|^{\beta}$ with $\alpha, \beta \geq 1$ satisfying $\alpha+\beta=N p /(N-p)$. We relate the number of solutions with the topology of the set where $V$ and $W$ attain it minimum values. We consider the subcritical case $\gamma=0$ and the critical case $\gamma=1$. In the proofs we apply Ljusternik-Schnirelmann theory.
\end{abstract}

Mathematics Subject Classification (2000). Primary 35J50; Secondary 35B33, 58E05.

Keywords: Quasilinear Schrödinger equation, Ljusternik-Schnirelmann theory, positive solutions, critical problems.

\section{Introduction}

In the first part of this paper we are concerned with the existence of multiple solutions for the quasilinear system

$$
\left\{\begin{array}{l}
-\varepsilon^{p} \Delta_{p} u+V(z) u^{p-1}=Q_{u}(u, v) \text { in } \mathbb{R}^{N}, \\
-\varepsilon^{p} \Delta_{p} v+W(z) v^{p-1}=Q_{v}(u, v) \text { in } \mathbb{R}^{N}, \\
u, v \in W^{1, p}\left(\mathbb{R}^{N}\right), \quad u(z), v(z)>0 \text { for all } z \in \mathbb{R}^{N},
\end{array}\right.
$$

where $\varepsilon>0,2 \leq p<N$ and $\Delta_{p} u=\operatorname{div}\left(|\nabla u|^{p-2} \nabla u\right)$ is the $p$-Laplacian operator. In order to make precise assumptions on the continuous potentials $V$ and $W$ we define

$$
\begin{aligned}
V_{0} & :=\inf _{x \in \mathbb{R}^{N}} V(x), \quad W_{0}:=\inf _{x \in \mathbb{R}^{N}} W(x), \\
V_{\infty} & :=\liminf _{|x| \rightarrow+\infty} V(x) \quad \text { and } \quad W_{\infty}:=\liminf _{|x| \rightarrow+\infty} W(x) .
\end{aligned}
$$

The second author was partially supported by FEMAT-DF 
and suppose that $V$ and $W$ satisfy

$\left(H_{0}\right) V_{0}=W_{0}>0$ and the set

$$
M:=\left\{x \in \mathbb{R}^{N}: V(x)=W(x)=V_{0}\right\}
$$

is nonempty.

$\left(H_{1}\right) V_{0}<\max \left\{V_{\infty}, W_{\infty}\right\}$.

Setting $R_{+}^{2}:=(0, \infty) \times(0, \infty)$, we can state our hypothesis on $Q \in C^{2}\left(\mathbb{R}_{+}^{2}, \mathbb{R}\right)$ in the following way.

$\left(Q_{0}\right)$ there exits $p<q<p^{*}:=N p /(N-p)$ such that

$$
Q(t u, t v)=t^{q} Q(u, v) \quad \text { for all } t>0,(u, v) \in \mathbb{R}_{+}^{2} .
$$

$\left(Q_{1}\right)$ there exists $C>0$ such that

$$
\left|Q_{u}(u, v)\right|+\left|Q_{v}(u, v)\right| \leq C\left(u^{q-1}+v^{q-1}\right) \quad \text { for all }(u, v) \in \mathbb{R}_{+}^{2} .
$$

$\left(Q_{2}\right) Q_{u}(0,1)=0, Q_{v}(1,0)=0$.

$\left(Q_{3}\right) Q_{u}(1,0)=0, Q_{v}(0,1)=0$.

$\left(Q_{4}\right) Q_{u v}(u, v)>0$ for all $(u, v) \in \mathbb{R}_{+}^{2}$.

We recall that, if $Y$ is a closed set of a topological space $X$, $\operatorname{cat}_{X}(Y)$ is the Ljusternik-Schnirelmann category of $Y$ in $X$, namely the least number of closed and contractible sets in $X$ which cover $Y$. We denote by

$$
M_{\delta}:=\left\{x \in \mathbb{R}^{N}: \operatorname{dist}(x, M) \leq \delta\right\}
$$

the closed $\delta$-neighborhood of $M$, and we shall prove the following multiplicity result.

Theorem 1.1. Suppose that $\left(H_{0}\right)-\left(H_{1}\right)$ and $\left(Q_{0}\right)-\left(Q_{4}\right)$ hold. Then, for any $\delta>0$ given, there exists $\varepsilon_{\delta}>0$ such that, for any $\varepsilon \in\left(0, \varepsilon_{\delta}\right)$, the system $\left(P_{\varepsilon}\right)$ has at least $\operatorname{cat}_{M_{\delta}}(M)$ solutions.

Note that the system $\left(P_{\varepsilon}\right)$ has a variational structure and therefore the solutions can be found as critical points of the functional

$$
\mathcal{I}_{\varepsilon}(u, v)=\frac{\varepsilon^{p}}{p} \int_{\mathbb{R}^{N}}\left(|\nabla u|^{p}+|\nabla v|^{p}+V(x)|u|^{p}+W(x)|v|^{p}\right) \mathrm{d} x-\int_{\mathbb{R}^{\mathbb{N}}} Q(u, v) \mathrm{d} x
$$

defined on an appropriated subspace of $W^{1, p}\left(\mathbb{R}^{N}\right) \times W^{1, p}\left(\mathbb{R}^{N}\right)$. In order to obtain such critical points we use a technique introduced by Benci and Cerami [7], which consists in making precise comparisons between the category of some 
sublevel sets of $\mathcal{I}$ and the category of the set $M$. This kind of argument for a scalar Schrödinger equation has appeared in [11]. Since we are intending to apply Ljusternik-Schnirelmann theory, we need to prove some compactness property for the functional $\mathcal{I}$. Following the ideas of $[28,11]$, we prove that the levels of compactness are strongly related with the behavior of the potentials $V(x)$ and $W(x)$ at infinity.

In the second part of the paper we deal with a critical version of $\left(P_{\varepsilon}\right)$, namely the problem

$\left(C P_{\varepsilon}\right), \quad\left\{\begin{array}{l}-\varepsilon^{p} \Delta_{p} u+V(z) u^{p-1}=Q_{u}(u, v)+\alpha|u|^{\alpha-2} u|v|^{\beta} \text { in } \mathbb{R}^{N}, \\ -\varepsilon^{p} \Delta_{p} v+W(z) v^{p-1}=Q_{v}(u, v)+\beta|u|^{\alpha}|v|^{\beta-2} v \text { in } \mathbb{R}^{N}, \\ u, v \in W^{1, p}\left(\mathbb{R}^{N}\right), \quad u(z), v(z)>0 \text { for all } z \in \mathbb{R}^{N},\end{array}\right.$

where the coefficients $\alpha, \beta \geq 1$ are such that the sum $\alpha+\beta$ is equal to the critical Sobolev exponent $p^{*}$. In order to deal with the critical growth of the nonlinearity we assume the same technical condition of [25], namely

$\left(Q_{5}\right) Q(u, v) \geq \lambda u^{\widetilde{\alpha}} v^{\widetilde{\beta}}$ for all $(u, v) \in \mathbb{R}_{+}^{2}$, with $1<\widetilde{\alpha}, \widetilde{\beta}<p^{*}, \widetilde{\alpha}+\widetilde{\beta}=q_{1} \in\left(p, p^{*}\right)$ and $\lambda$ satisfying

$\left(Q_{5} a\right) \lambda>0$ if either $N \geq p^{2}$, or $p<N<p^{2}$ and $p^{*}-p /(p-1)<q_{1}<p^{*}$, $\left(Q_{5} b\right) \lambda$ is sufficiently large if $p<N<p^{2}$ and $p<q_{1} \leq p^{*}-p /(p-1)$.

The critical version of Theorem 1.1 can be stated as follows.

Theorem 1.2. Suppose that $\left(H_{0}\right)-\left(H_{1}\right)$ and $\left(Q_{0}\right)-\left(Q_{5}\right)$ hold. If $\alpha, \beta \in\left[1, p^{*}\right)$ satisfy $\alpha+\beta=p^{*}$ then, for any $\delta>0$ given, there exists $\varepsilon_{\delta}>0$ such that, for any $\varepsilon \in\left(0, \varepsilon_{\delta}\right)$, the system $\left(C P_{\varepsilon}\right)$ has at least cat $_{M_{\delta}}(M)$ solutions.

The proof of Theorem 1.2 follows the same lines of the subcritical case. However, this new problem has an extra difficult when compared with the subcritical one. This occurs because the level of non-compactness is affected by the critical growth of the nonlinearity. This problem is overcame by using the ideas of Brezis and Nirenberg [10], some adaptations of the calculations performed in [25], besides the paper [2], where it is proved that the number

$$
\widetilde{S}(\alpha, \beta):=\inf _{u, v \in W^{1, p}\left(\mathbb{R}^{N}\right) \backslash\{0\}} \frac{\int_{\mathbb{R}^{N}}|\nabla u|^{p} \mathrm{~d} x+\int_{\mathbb{R}^{N}}|\nabla v|^{p} \mathrm{~d} x}{\left(\int_{\mathbb{R}^{N}}|u|^{\alpha}|v|^{\beta} \mathrm{d} x\right)^{p / p^{*}}}
$$

plays an important role when dealing with critical systems with coupled critical part as in $\left(C P_{\varepsilon}\right)$. 
The semilinear scalar case of the problems considered here are related with the equation

$$
-\frac{\varepsilon^{2}}{2 m} \Delta u+a(z) u=\gamma|u|^{r-2} u \text { in } \mathbb{R}^{N}
$$

which naturally appears when we look for standing wave solutions $\psi(z, t):=$ $\exp \left(-i \varepsilon^{-1} t\right) u(z)$ for the nonlinear Schrödinger equation

$$
i \varepsilon \frac{\partial \psi}{\partial t}=-\frac{\varepsilon^{2}}{2 m} \Delta \psi+a(z) \psi-\gamma|u|^{r-2} u \text { in } \mathbb{R}^{N}
$$

where $\varepsilon, m$ and $\gamma$ are positive constants and $r>1$. There is vast literature concerning the existence and multiplicity of solutions for the equation (1.1) (see $[19,26,27,32,13,14,4,33,15]$ and references there in). In particular, we would like to cite the paper of Rabinowitz [28] where it is supposed that the potential $a$ verified

$$
0<\inf _{x \in \mathbb{R}^{N}} a(x)<\liminf _{|x| \rightarrow \infty} a(x) .
$$

The conditions $\left(H_{0}\right)-\left(H_{1}\right)$ are clearly inspired by the above hypotheses. However, in our multiplicity results, we allow the situation where $V_{0}=\min \left\{V_{\infty}, W_{\infty}\right\}$ provided $V_{0}<\max \left\{V_{\infty}, W_{\infty}\right\}$.

Some existence results about systems can be found in $[17,16,9,3,21,22,23]$. The hypothesis on $Q$ used here have appeared in [17], where some properties and examples of nonlinearities $Q$ verifying $\left(Q_{0}\right)-\left(Q_{4}\right)$ were presented. In [3] the authors considered the subcritical problem $\left(P_{\varepsilon}\right)$ with $p=2$. In order to explain their results we define, for any fixed $\xi \in \mathbb{R}^{N}$, the functional $\mathcal{I}_{\xi}: H^{1}\left(\mathbb{R}^{N}\right) \times$ $H^{1}\left(\mathbb{R}^{N}\right) \rightarrow \mathbb{R}$ as

$$
\mathcal{I}_{\xi}(u, v)=\frac{1}{2} \int_{\mathbb{R}^{N}}\left(|\nabla u|^{2}+|\nabla v|^{2}+V(\xi) u^{2}+W(\xi) v^{2}\right) \mathrm{d} x-\int_{\mathbb{R}^{\mathbb{N}}} Q(u, v) \mathrm{d} x
$$

and the ground state function $\mathcal{C}: \mathbb{R}^{N} \rightarrow \mathbb{R}$ by setting

$$
\mathcal{C}(\xi)=\inf _{(u, v) \neq(0,0)} \max _{t \geq 0} \mathcal{I}_{\xi}(t u, t v)
$$

They proved results concerning the existence and concentration behavior, for $\varepsilon>0$ small, of ground state solutions of $\left(P_{\varepsilon}\right)$ provided $V_{0}>0, W_{0}>0$ and

$$
\inf _{\xi \in \mathbb{R}^{N}} \mathcal{C}(\xi)<\mathcal{C}_{\infty}
$$

Here $\mathcal{C}_{\infty}$ denotes the ground state level of the functional $\mathcal{I}_{\infty}$ obtained by replacing $V(\xi)$ and $W(\xi)$ by $V_{\infty}$ and $W_{\infty}$, respectively, in the definition of $\mathcal{I}_{\xi}$. Note that the above assumption is weaker than $\left(H_{0}\right)-\left(H_{1}\right)$. We do not know if our multiplicity results can be proved in this weaker setting. 
Some multiplicity results for scalar Schrödinger equations via LjusternikSchnirelmann theory can be found in $[6,12,11]$. For systems, we can cite three recent papers $[5,18,31]$, which deal only with the semilinear case $p=2$ and subcritical nonlinearities. Among them, the most related with our results are $[18,31]$. In [18] it was supposed $V \equiv W$ and that $Q$ is a coupled power. In [31] the authors studied a Hamiltonian version of the problem $\left(P_{\varepsilon}\right)$ with $V \equiv W$. Note that our condition $\left(H_{0}\right)-\left(H_{1}\right)$ are weaker than $V \equiv W$.

In view of the results presented in $[9,5,3,31]$, it is natural to ask if we can obtain multiplicity results for the quasilinear systems $\left(P_{\varepsilon}\right)$ and $\left(C P_{\varepsilon}\right)$. In this paper, we present a positive answer for this question. Our results also complement most of the aforementioned works, since we consider multiplicity of solutions, the quasilinear problem and both, subcritical and critical growth. Finally we would like to emphasize two points: first that, although we deal with the quasilinear case, our result seem to be new even in the semilinear case $p=2$; secondly that, to the best of our knowledge, there is no results concerning multiplicity of solutions for systems with critical growth via Ljusternik-Schnirelmann theory.

The paper is organized as follows. In Section 2 we present the abstract framework of the subcritical case besides the compactness properties of the functional associated to $\left(P_{\varepsilon}\right)$. Section 3 is devoted to the proof of Theorem 1.1, while the multiplicity result in the critical case is proved in Section 4 .

\section{Variational framework}

Since we are interested in positive solutions, we extend the function $Q$ to the whole $\mathbb{R}^{2}$ by setting $Q(u, v)=0$ if $u \leq 0$ or $v \leq 0$. For simplicity, we write only $\int u$ instead of $\int_{\mathbb{R}^{N}} u(x) \mathrm{d} x$. We also note that, since $Q$ is $q$-homogeneous, the following holds

$$
q Q(s, t)=s Q_{s}(s, t)+t Q_{t}(s, t) \text { for all }(s, t) \in \mathbb{R}^{2} .
$$

Hereafter, we will work with the following system equivalent to $\left(P_{\varepsilon}\right)$, which is obtained under the change of variables $z \mapsto \varepsilon x$

$$
\left\{\begin{array}{l}
-\Delta_{p} u+V(\varepsilon x)|u|^{p-2} u=Q_{u}(u, v) \text { in } \mathbb{R}^{N}, \\
-\Delta_{p} v+W(\varepsilon x)|v|^{p-2} v=Q_{v}(u, v) \text { in } \mathbb{R}^{N}, \\
u(x), v(x)>0 \text { for all } x \in \mathbb{R}^{N} .
\end{array}\right.
$$

For any $\varepsilon>0$, we consider the Sobolev space

$$
X_{\varepsilon}:=\left\{(u, v) \in W^{1, p}\left(\mathbb{R}^{N}\right) \times W^{1, p}\left(\mathbb{R}^{N}\right): \int\left(V(\varepsilon x)|u|^{p}+W(\varepsilon x)|v|^{p}\right)<\infty\right\}
$$

endowed with the norm

$$
\|(u, v)\|_{\varepsilon}:=\left\{\int\left(|\nabla u|^{p}+|\nabla v|^{p}\right)+\int\left(V(\varepsilon x)|u|^{p}+W(\varepsilon x)|v|^{p}\right)\right\}^{1 / p} .
$$


The growth condition $\left(Q_{1}\right)$ imply that, for some other constant $C>0$,

$$
|Q(u, v)| \leq C\left(|u|^{q}+|v|^{q}\right) \text { for all }(u, v) \in \mathbb{R}^{2} .
$$

Hence, the weak solutions of the system $\left(\widehat{P}_{\varepsilon}\right)$ are related with the critical points of the functional $I_{\varepsilon}: X_{\varepsilon} \rightarrow \mathbb{R}$ given by

$$
I_{\varepsilon}(u, v):=\frac{1}{p}\|(u, v)\|_{\varepsilon}^{p}-\int Q(u, v) .
$$

We introduce the Nehari manifold of $I_{\varepsilon}$ by setting

$$
\mathcal{N}_{\varepsilon}:=\left\{(u, v) \in X_{\varepsilon} \backslash\{(0,0)\}:\left\langle I_{\varepsilon}^{\prime}(u, v),(u, v)\right\rangle=0\right\}
$$

and define the minimax $c_{\varepsilon}$ as being

$$
c_{\varepsilon}:=\inf _{(u, v) \in \mathcal{N}_{\varepsilon}} I_{\varepsilon}(u, v) .
$$

In what follows, we present some properties of $c_{\varepsilon}$ and $\mathcal{N}_{\varepsilon}$. Its proofs can de done as in [34, Chapter 4]. First of all, we note that there exists $r>0$, independent of $\varepsilon$, such that

$$
\|(u, v)\|_{\varepsilon} \geq r>0 \quad \text { for any } \varepsilon>0,(u, v) \in \mathcal{N}_{\varepsilon} .
$$

Since $I_{\varepsilon}$ satisfies Mountain Pass geometry, we can use the homogeneity of $Q$ to prove that $c_{\varepsilon}$ can be alternatively characterized by

$$
c_{\varepsilon}=\inf _{\gamma \in \Gamma_{\varepsilon}} \max _{t \in[0,1]} I_{\varepsilon}(\gamma(t))=\inf _{(u, v) \in X_{\varepsilon} \backslash\{(0,0)\}} \max _{t \geq 0} I_{\varepsilon}(t u, t v)>0,
$$

where $\Gamma_{\varepsilon}:=\left\{\gamma \in C\left([0,1], X_{\varepsilon}\right): \gamma(0)=0, I_{\varepsilon}(\gamma(1))<0\right\}$. Moreover, for any $(u, v) \neq(0,0)$, there exists a unique $\bar{t}>0$ such that $(\bar{t} u, \bar{t} v) \in \mathcal{N}_{\varepsilon}$. The maximum of the function $t \mapsto I_{\varepsilon}(t u, t v)$ for $t \geq 0$ is achieved at $t=\bar{t}$.

\subsection{The autonomous problem}

As we will see, it is important to compare the number $c_{\varepsilon}$ with other one, which is related with the following autonomous problem

$$
\left\{\begin{array}{l}
-\Delta_{p} u+V_{0}|u|^{p-2} u=Q_{u}(u, v) \text { in } \mathbb{R}^{N}, \\
-\Delta_{p} v+W_{0}|v|^{p-2} v=Q_{v}(u, v) \text { in } \mathbb{R}^{N}, \\
u(x), v(x)>0 \text { for all } x \in \mathbb{R}^{N} .
\end{array}\right.
$$

If we denote by $X_{0}$ the space $W^{1, p}\left(\mathbb{R}^{N}\right) \times W^{1, p}\left(\mathbb{R}^{N}\right)$ endowed with the norm

$$
\|(u, v)\|_{0}:=\left\{\int\left(|\nabla u|^{p}+|\nabla v|^{p}\right)+\int\left(V_{0}|u|^{p}+W_{0}|v|^{p}\right)\right\}^{1 / p}
$$


we have an associated functional $I_{0}: X_{0} \rightarrow \mathbb{R}$ given by

$$
I_{0}(u, v):=\|(u, v)\|_{0}^{p}-\int Q(u, v),
$$

and the minimax level

$$
c_{0}:=\inf _{(u, v) \in \mathcal{N}_{0}} I_{0}(u, v)=\inf _{(u, v) \in X_{0} \backslash\{(0,0)\}} \max _{t \geq 0} I_{0}(t u, t v)>0,
$$

where $\mathcal{N}_{0}:=\left\{(u, v) \in X_{0} \backslash\{(0,0)\}:\left\langle I_{0}^{\prime}(u, v),(u, v)\right\rangle=0\right\}$ is the Nehari manifold of $I_{0}$.

We shall prove that $c_{0}$ is achieved. For that, we need the following technical lemma, whose proof was inspired by [3].

Lemma 2.1. Let $\left(\left(u_{n}, v_{n}\right)\right) \subset X_{0}$ be a bounded sequence such that $I_{0}^{\prime}\left(u_{n}, v_{n}\right) \rightarrow 0$. Then we have either

(i) $\left\|\left(u_{n}, v_{n}\right)\right\|_{0} \rightarrow 0$, or

(ii) there exists a sequence $\left(y_{n}\right) \subset \mathbb{R}^{N}$ and constants $R, \gamma>0$ such that

$$
\liminf _{n \rightarrow \infty} \int_{B_{R}\left(y_{n}\right)}\left(\left|u_{n}\right|^{p}+\left|v_{n}\right|^{p}\right) \geq \gamma>0 .
$$

Proof. Suppose that (ii) does not hold. Then we have

$$
\lim _{n \rightarrow+\infty} \sup _{y \in \mathbb{R}^{N}} \int_{B_{R}(y)}\left|u_{n}\right|^{p}=0 \text { and } \lim _{n \rightarrow+\infty} \sup _{y \in \mathbb{R}^{N}} \int_{B_{R}(y)}\left|v_{n}\right|^{p}=0,
$$

for any $R>0$. This and [24, Lemma I.1] imply that

$$
\lim _{n \rightarrow \infty} \int\left|u_{n}\right|^{q}=0 \quad \text { and } \quad \lim _{n \rightarrow \infty} \int\left|v_{n}\right|^{q}=0 .
$$

Thus, we can use $(2.2)$ to conclude that $\int Q\left(u_{n}, v_{n}\right) \rightarrow 0$. Since $\left(\left(u_{n}, v_{n}\right)\right)$ is bounded we have that $\left\langle I_{0}^{\prime}\left(u_{n}, v_{n}\right),\left(u_{n}, v_{n}\right)\right\rangle \rightarrow 0$. This and (2.1) provide

$$
\left\|\left(u_{n}, v_{n}\right)\right\|_{0}^{p}=q \int Q\left(u_{n}, v_{n}\right)=o_{n}(1),
$$

where $o_{n}(1)$ denotes a quantity approaching zero as $n \rightarrow \infty$. Hence (i) holds and we conclude the proof.

Proposition 2.2. The problem $(A)$ has a weak solution. 
Proof. Since $I_{0}$ has the Mountain Pass geometry, there exits $\left(\left(u_{n}, v_{n}\right)\right) \subset X_{0}$ such that

$$
I_{0}\left(u_{n}, v_{n}\right) \rightarrow c_{0} \text { and } I_{0}^{\prime}\left(u_{n}, v_{n}\right) \rightarrow 0
$$

In view of $(2.1)$, we have that

$$
\begin{aligned}
c_{0}+o_{n}(1)\left\|\left(u_{n}, v_{n}\right)\right\|_{0} & =I_{0}\left(u_{n}, v_{n}\right)-\frac{1}{q}\left\langle I_{0}^{\prime}\left(u_{n}, v_{n}\right),\left(u_{n}, v_{n}\right)\right\rangle \\
& =\left(\frac{1}{p}-\frac{1}{q}\right)\left\|\left(u_{n}, v_{n}\right)\right\|_{0}^{p} .
\end{aligned}
$$

Since $p \geq 2$, it follows from the above expression that $\left(\left(u_{n}, v_{n}\right)\right)$ is bounded in $X_{0}$. Hence, up to a subsequence, $\left(u_{n}, v_{n}\right) \rightarrow(u, v)$ weakly in $X_{0}$ and $u_{n}(x) \rightarrow u(x)$, $v_{n}(x) \rightarrow v(x)$ a.e. in $\mathbb{R}^{N}$. By adapting standard calculations [35] we can obtain a subsequence, still denoted by $\left(\left(u_{n}, v_{n}\right)\right)$, such that

$$
\begin{array}{ll}
\nabla u_{n}(x) \rightarrow \nabla u(x), \nabla v_{n}(x) \rightarrow \nabla v(x) & \text { a.e. } x \in \mathbb{R}^{N}, \\
\left|\nabla u_{n}\right|^{p-2} \frac{\partial u_{n}}{\partial x_{i}} \rightarrow|\nabla u|^{p-2} \frac{\partial u}{\partial x_{i}} & \text { weakly in }\left(L^{p}\left(\mathbb{R}^{N}\right)\right)^{*}, \\
\left|\nabla v_{n}\right|^{p-2} \frac{\partial v_{n}}{\partial x_{i}} \rightarrow|\nabla v|^{p-2} \frac{\partial v}{\partial x_{i}} & \text { weakly in }\left(L^{p}\left(\mathbb{R}^{N}\right)\right)^{*},
\end{array}
$$

for all $1 \leq i \leq N$. The weak convergence of $\left(\left(u_{n}, v_{n}\right)\right)$, the above expression and $\left(Q_{1}\right)$ imply that $I_{0}^{\prime}(u, v)=0$.

Suppose first that $u \not \equiv 0$ and $v \not \equiv 0$. Then, if we denote by $u^{-}=\max \{-u, 0\}$ and $v^{-}=\max \{-v, 0\}$ the negative part of $u$ and $v$, respectively, we get

$$
\begin{aligned}
0 & =\left\langle I_{0}^{\prime}(u, v),\left(u^{-}, v^{-}\right)\right\rangle \\
& =\left\|\left(u^{-}, v^{-}\right)\right\|_{0}^{p}-\int\left(Q_{u}(u, v) u^{-}+Q_{v}(u, v) v^{-}\right)=\left\|\left(u^{-}, v^{-}\right)\right\|_{0}^{p},
\end{aligned}
$$

where we have used in the last equality that $Q_{u} \equiv 0$ on $(-\infty, 0) \times \mathbb{R}$ and $Q_{v} \equiv 0$ on $\mathbb{R} \times(-\infty, 0)$. It follows from the above expression that $u, v \geq 0$ in $\mathbb{R}^{N}$. Since $Q$ is $q$-homogeneous, we have that $\nabla Q$ is $(q-1)$-homogeneous. Thus, we can use conditions $\left(Q_{3}\right),\left(Q_{4}\right)$ and the Mean Value Theorem to prove that $Q_{u}$ and $Q_{v}$ are nonnegative functions. Moreover, adapting arguments from [20, Theorem 1.11] we conclude that $u, v \in L^{\infty}\left(\mathbb{R}^{N}\right) \cap C_{\text {loc }}^{1, \alpha}\left(\mathbb{R}^{N}\right)$ for some $0<\alpha<1$. Since we already known that $u, v \geq 0$, we can use Harnack's inequality [30, Theorem 1.1] to conclude that $u$ and $v$ are positive functions.

We claim that $I_{0}(u, v)=c_{0}$. Indeed, since $(u, v) \in \mathcal{N}_{0}$, we can use $(2.1)$ and Fatou's lemma to get 


$$
\begin{aligned}
c_{0} & \leq I_{0}(u, v)=\frac{q-p}{p} \int Q(u, v) \\
& \leq \liminf _{n \rightarrow \infty} \frac{q-p}{p} \int Q\left(u_{n}, v_{n}\right) \\
& =\liminf _{n \rightarrow \infty}\left(I_{0}\left(u_{n}, v_{n}\right)-\frac{1}{p}\left\langle I_{0}^{\prime}\left(u_{n}, v_{n}\right),\left(u_{n}, v_{n}\right)\right\rangle\right)=c_{0} .
\end{aligned}
$$

Thus, $(u, v)$ is the required solution.

We now consider the case $u \equiv 0$ or $v \equiv 0$. If $u \equiv 0$ then we can use $\left\langle I_{0}^{\prime}(u, v),(u, v)\right\rangle=0$ and $(2.1)$ to get

$$
\|v\|_{0}^{p}=\int Q_{u}(0, v) v=q \int Q(0, v)=0
$$

and therefore $v \equiv 0$. Analogously, $v \equiv 0$ implies $u \equiv 0$. Hence, if $u \equiv 0$ or $v \equiv 0$, we must have $(u, v)=(0,0)$. Recalling that $c_{0}>0$ and $I_{0}$ is continuous, we conclude that $\left\|\left(u_{n}, v_{n}\right)\right\|_{0}$ cannot goes to zero. The above lemma provides a sequence $\left(y_{n}\right) \subset \mathbb{R}^{N}$ and constants $R, \gamma>0$ such that

$$
\liminf _{n \rightarrow \infty} \int_{B_{R}\left(y_{n}\right)}\left(\left|u_{n}\right|^{p}+\left|v_{n}\right|^{p}\right) \geq \gamma>0 .
$$

If we define $\left(\widetilde{u}_{n}(x), \widetilde{v}_{n}(x)\right):=\left(u_{n}\left(x+y_{n}\right), v_{n}\left(x+y_{n}\right)\right)$ we can use the invariance of $\mathbb{R}^{N}$ by translations to conclude that $I_{0}\left(\tilde{u}_{n}, \tilde{v}_{n}\right) \rightarrow c_{0}$ and $I_{0}^{\prime}\left(\tilde{u}_{n}, \tilde{v}_{n}\right) \rightarrow 0$. Moreover, up to a subsequence, $\left(\tilde{u}_{n}, \tilde{v}_{n}\right) \rightarrow(\tilde{u}, \tilde{v})$ weakly in $X_{0}$ and $\tilde{u}_{n} \rightarrow \tilde{u}, \tilde{v}_{n} \rightarrow$ $\tilde{v}$ strongly in $L^{p}\left(B_{R}(0)\right)$, with $(\tilde{u}, \tilde{v})$ being a critical point of $I_{0}$. Since

$$
\int_{B_{R}(0)}\left(|\tilde{u}|^{p}+|\tilde{v}|^{p}\right)=\liminf _{n \rightarrow \infty} \int_{B_{R}\left(y_{n}\right)}\left(\left|u_{n}\right|^{p}+\left|v_{n}\right|^{p}\right) \geq \gamma>0,
$$

we conclude that $\tilde{u} \not \equiv 0$ or $\tilde{v} \not \equiv 0$. Hence, as at the beginning of the paragraph, we can conclude that both $\tilde{u}$ and $\tilde{v}$ are nonzero. Arguing as in the first part of the proof we conclude that $(\tilde{u}, \tilde{v})$ is the desired solution.

\subsection{The Palais-Smale condition}

We start this subsection by recalling the definition of the Palais-Smale condition. So, let $E$ be a Banach space, $\mathcal{V}$ be a $C^{1}$-manifold of $E$ and $I: E \rightarrow \mathbb{R}$ a $C^{1}$ functional. We say that $\left.I\right|_{\mathcal{V}}$ satisfies the Palais-Smale condition at level $d\left((\mathrm{PS})_{d}\right.$ for short) if any sequence $\left(u_{n}\right) \subset \mathcal{V}$ such that $I\left(u_{n}\right) \rightarrow d$ and $\left\|I^{\prime}\left(u_{n}\right)\right\|_{*} \rightarrow 0$ contains a convergent subsequence. Here, we are denoting by $\left\|I^{\prime}(u)\right\|_{*}$ the norm of the derivative of $I$ restricted to $\mathcal{V}$ at the point $u$

If $\max \left\{V_{\infty}, W_{\infty}\right\}<\infty$, we define the limit functional $I_{\infty}: X_{0} \rightarrow \mathbb{R}$ as

$$
I_{\infty}(u, v):=\frac{1}{p} \int\left(|\nabla u|^{p}+|\nabla v|^{p}+V_{\infty}|u|^{p}+W_{\infty}|v|^{p}\right)-\int Q(u, v),
$$


and denote by $c_{\infty}$ the ground state level of $I_{\infty}$, namely

$$
c_{\infty}:=\inf _{(u, v) \in \mathcal{N}_{\infty}} I_{\infty}(u, v)=\inf _{(u, v) X_{0} \backslash\{(0,0)\}} \max _{t \geq 0} I_{\infty}(t u, t v)>0,
$$

where $\mathcal{N}_{\infty}:=\left\{(u, v) \in X_{0} \backslash\{(0,0)\}:\left\langle I_{\infty}^{\prime}(u, v),(u, v)\right\rangle=0\right\}$. If $\max \left\{V_{\infty}, W_{\infty}\right\}=$ $\infty$, we set $c_{\infty}:=\infty$.

We state below our compactness result for $I_{\varepsilon}$.

Proposition 2.3. The functional $I_{\varepsilon}$ constrained to $\mathcal{N}_{\varepsilon}$ satisfies the $(\mathrm{PS})_{d}$ condition at any level $d<c_{\infty}$.

For the proof of this proposition we need the following auxiliar result.

Lemma 2.4. Suppose that $\max \left\{V_{\infty}, W_{\infty}\right\}<\infty$. Let $\left(\left(u_{n}, v_{n}\right)\right) \subset X_{\varepsilon}$ be a $(\mathrm{PS})_{d}$ sequence for $I_{\varepsilon}$ such that $\left(u_{n}, v_{n}\right) \rightarrow(0,0)$ weakly in $X_{\varepsilon}$. If $\left(u_{n}, v_{n}\right) \nrightarrow(0,0)$ strongly in $X_{\varepsilon}$, then $d \geq c_{\infty}$.

Proof. Let $\left(t_{n}\right) \subset(0,+\infty)$ be such that $\left(t_{n} u_{n}, t_{n} v_{n}\right) \in \mathcal{N}_{\infty}$. We start by proving that

$$
t_{0}:=\limsup _{n \rightarrow \infty} t_{n} \leq 1 .
$$

Arguing by contradiction, we suppose that there exists $\lambda>0$ and a subsequence, still denoted by $\left(t_{n}\right)$, such that

$$
t_{n} \geq 1+\lambda \text { for all } n \in \mathbb{N} \text {. }
$$

Since $\left(\left(u_{n}, v_{n}\right)\right)$ is bounded in $X_{\varepsilon}$, we have that $\left\langle I_{\varepsilon}^{\prime}\left(u_{n}, v_{n}\right),\left(u_{n}, v_{n}\right)\right\rangle \rightarrow 0$, that is

$$
\int\left(\left|\nabla u_{n}\right|^{p}+\left|\nabla v_{n}\right|^{p}+V(\varepsilon x)\left|u_{n}\right|^{p}+W(\varepsilon x)\left|v_{n}\right|^{p}\right)=q \int Q\left(u_{n}, v_{n}\right)+o_{n}(1) .
$$

Moreover, recalling that $\left(t_{n}\left(u_{n}, v_{n}\right)\right) \subset \mathcal{N}_{\infty}$, we get

$$
\int\left(\left|\nabla u_{n}\right|^{p}+\left|\nabla v_{n}\right|^{p}+V_{\infty}\left|u_{n}\right|^{p}+W_{\infty}\left|v_{n}\right|^{p}\right)=q t_{n}^{q-p} \int Q\left(u_{n}, v_{n}\right) .
$$

These two equalities imply that

$$
\begin{aligned}
q\left(t_{n}^{q-p}-1\right) \int Q\left(u_{n}, v_{n}\right)= & \int\left(V_{\infty}-V(\varepsilon x)\right)\left|u_{n}\right|^{p} \\
& +\int\left(W_{\infty}-W(\varepsilon x)\right)\left|v_{n}\right|^{p}+o_{n}(1) .
\end{aligned}
$$

Given $\zeta>0$ we take $R>0$ such that

$$
V(\varepsilon x) \geq V_{\infty}-\zeta, \quad W(\varepsilon x) \geq W_{\infty}-\zeta \text { for all }|x| \geq R .
$$


Since $\left(\left(u_{n}, v_{n}\right)\right) \rightarrow(0,0)$ in $W^{1, p}\left(B_{R}(0)\right) \times W^{1, p}\left(B_{R}(0)\right)$ we can use $(2.5),(2.6)$ and (2.7) to obtain

$$
q\left((1+\lambda)^{q-p}-1\right) \int Q\left(u_{n}, v_{n}\right) \leq q\left(t_{n}^{q-p}-1\right) \int Q\left(u_{n}, v_{n}\right) \leq \zeta C+o_{n}(1),
$$

where $C>0$ is such that $\left\|\left(u_{n}, v_{n}\right)\right\|_{\varepsilon} \leq C$.

Since $\left(u_{n}, v_{n}\right) \nrightarrow(0,0)$ we can argue as in the proof of Lemma 2.1 to obtain $\left(y_{n}\right) \subset \mathbb{R}^{N}$ and $R, \gamma>0$ such that

$$
\int_{B_{R}\left(y_{n}\right)}\left(\left|u_{n}\right|^{p}+\left|v_{n}\right|^{p}\right) \geq \gamma>0 .
$$

If we define $\left(\widetilde{u}_{n}(x), \widetilde{v}_{n}(x)\right):=\left(u_{n}\left(x+y_{n}\right), v_{n}\left(x+y_{n}\right)\right)$ we may suppose that, up to a subsequence,

$$
\begin{aligned}
& \left(\widetilde{u}_{n}, \widetilde{v}_{n}\right) \rightarrow(u, v) \text { weakly in } X_{\varepsilon}, \\
& \left(\widetilde{u}_{n}, \widetilde{v}_{n}\right) \rightarrow(u, v) \text { in } L^{p}\left(B_{R}(0)\right) \times L^{p}\left(B_{R}(0)\right), \\
& \left(\widetilde{u}_{n}(x), \widetilde{v}_{n}(x)\right) \rightarrow(u(x), v(x)) \text { for a.e. } x \in \mathbb{R}^{N},
\end{aligned}
$$

for some nonnegative functions $u, v$ with $I_{\varepsilon}^{\prime}(u, v)=0$. In view of (2.9) we have that $u \neq 0$ or $v \neq 0$. Hence, as in the proof of Proposition 2.2, $u$ and $v$ are positive in $\mathbb{R}^{N}$.

Letting $n \rightarrow \infty$ in (2.8) and using Fatou's lemma, we obtain

$$
0<q\left((1+\lambda)^{q-p}-1\right) \int Q(u, v) \leq \zeta C .
$$

Since $\zeta>0$ is arbitrary, we obtain a contradiction by taking $\zeta \rightarrow 0$. Hence, $t_{0} \leq 1$ as claimed.

Now, we divide the proof in two complementary cases.

Case $1 t_{0}<1$.

In this case we may suppose, without loss of generality, that $t_{n}<1$ for all $n \in \mathbb{N}$. Thus,

$$
\begin{aligned}
c_{\infty} & \leq I_{\infty}\left(\left(t_{n} u_{n}, t_{n} v_{n}\right)\right)-\frac{1}{p}\left\langle I_{\infty}^{\prime}\left(t_{n} u_{n}, t_{n} v_{n}\right),\left(t_{n} u_{n}, t_{n} v_{n}\right)\right\rangle \\
& =t_{n}^{q}\left(\frac{q-p}{p}\right) \int Q\left(u_{n}, v_{n}\right) \leq\left(\frac{q-p}{p}\right) \int Q\left(u_{n}, v_{n}\right) \\
& =I_{\varepsilon}\left(u_{n}, v_{n}\right)-\frac{1}{p}\left\langle I_{\varepsilon}^{\prime}\left(u_{n}, v_{n}\right),\left(u_{n}, v_{n}\right)\right\rangle=d+o_{n}(1) .
\end{aligned}
$$

Taking the limit we conclude that $d \geq c_{\infty}$.

Case $2 t_{0}=1$. 
Up to a subsequence, we may suppose that $t_{n} \rightarrow 1$. We first note that

$$
d+o_{n}(1) \geq c_{\infty}+I_{\varepsilon}\left(u_{n}, v_{n}\right)-I_{\infty}\left(t_{n} u_{n}, t_{n} v_{n}\right) .
$$

Given $\zeta>0$ we can use $\left(H_{1}\right)$ as in the beginning of the proof, the $q$-homogeneity of $Q$, the boundedness of $\left(\left(u_{n}, v_{n}\right)\right)$ and $t_{n} \rightarrow 1$ to estimate

$$
\begin{aligned}
I_{\varepsilon}\left(u_{n}, v_{n}\right)- & I_{\infty}\left(t_{n} u_{n}, t_{n} v_{n}\right)=\frac{\left(1-t_{n}^{p}\right)}{p} \int\left(\left|\nabla u_{n}\right|^{p}+\left|\nabla v_{n}\right|^{p}\right) \\
& +\frac{1}{p} \int\left(V(\varepsilon x)\left|u_{n}\right|^{p}+W(\varepsilon x)\left|v_{n}\right|^{p}\right)-\frac{t_{n}^{p}}{p} \int\left(V_{\infty}\left|u_{n}\right|^{p}+W_{\infty}\left|v_{n}\right|^{p}\right) \\
& +\left(t_{n}^{q}-1\right) \int Q\left(u_{n}, v_{n}\right) \\
\geq & o_{n}(1)-\zeta C .
\end{aligned}
$$

Hence

$$
d+o_{n}(1) \geq c_{\infty}-\zeta C+o_{n}(1)
$$

for any $\zeta>0$. By taking $n \rightarrow \infty$ and $\zeta \rightarrow 0$, we conclude that $d \geq c_{\infty}$.

Corollary 2.5. Suppose that $\max \left\{V_{\infty}, W_{\infty}\right\}=\infty$. If $\left(\left(u_{n}, v_{n}\right)\right) \subset X_{\varepsilon}$ is a (PS $)_{d}$ sequence for $I_{\varepsilon}$ such that $\left(u_{n}, v_{n}\right) \rightarrow(0,0)$ weakly in $X_{\varepsilon}$, then $\left(u_{n}, v_{n}\right) \rightarrow(0,0)$ strongly in $X_{\varepsilon}$.

Proof. For any $(a, b) \in \mathbb{R}_{+}^{2}$ we can define

$$
c_{(a, b)}:=\inf _{(u, v) \in X_{0} \backslash\{(0,0)\}} \max _{t \geq 0} I_{(a, b)}(t u, t v),
$$

where

$$
I_{(a, b)}(u, v):=\frac{1}{p} \int\left(|\nabla u|^{p}+|\nabla v|^{p}+a|u|^{p}+b|v|^{p}\right)-\int Q(u, v) .
$$

The same calculations performed in Proposition 2.2 show that, for any fixed $(a, b) \in \mathbb{R}_{+}^{2}$, the number $c_{(a, b)}$ is achieved by a pair $(u, v)$ of positive functions. It is easy to check that, if $a>a^{\prime}$, then $c_{(a, b)}>c_{\left(a^{\prime}, b\right)}$. Moreover, $\lim _{a^{2}+b^{2} \rightarrow \infty}$ $c_{(a, b)}=\infty$.

Since $\max \left\{V_{\infty}, W_{\infty}\right\}=\infty$ we can take $(a, b) \in \mathbb{R}_{+}^{2}$ in such way that $c_{(a, b)}>d$ and, for any given $\zeta>0$, there exists $R>0$ such that

$$
V(\varepsilon x) \geq a-\zeta, \quad W(\varepsilon x) \geq b-\zeta \text { for all }|x| \geq R .
$$

For example, if $W_{\infty}<\infty$, we take $b=W_{\infty}$ and $a>0$ large. If $V_{\infty}=W_{\infty}=\infty$ we take both $a$ and $b$ large.

Now, suppose by contradiction that $\left(u_{n}, v_{n}\right) \nrightarrow(0,0)$. Then we can use (2.10) and argue as in the proof of Lemma 2.4 to conclude that $d \geq c_{(a, b)}$, which does not make sense. Hence $\left(u_{n}, v_{n}\right) \rightarrow(0,0)$ and the corollary is proved. 
We are now able to prove our compactness result.

Proof of Proposition 2.3. Let $\left(\left(u_{n}, v_{n}\right)\right) \subset \mathcal{N}_{\varepsilon}$ be such that

$$
I_{\varepsilon}\left(u_{n}, v_{n}\right) \rightarrow d \text { and }\left\|I_{\varepsilon}^{\prime}\left(u_{n}, v_{n}\right)\right\|_{*} \rightarrow 0 .
$$

Then there exists a sequence $\left(\lambda_{n}\right) \subset \mathbb{R}$ such that

$$
I_{\varepsilon}^{\prime}\left(u_{n}, v_{n}\right)=\lambda_{n} F_{\varepsilon}^{\prime}\left(u_{n}, v_{n}\right)+o_{n}(1),
$$

where $F_{\varepsilon}:(u, v) \mapsto\|(u, v)\|_{\varepsilon}^{p}-q \int Q(u, v)$. Hence

$$
\begin{aligned}
0=\left\langle I_{\varepsilon}^{\prime}\left(u_{n}, v_{n}\right),\left(u_{n}, v_{n}\right)\right\rangle & =\lambda_{n}\left\langle F_{\varepsilon}^{\prime}\left(u_{n}, v_{n}\right),\left(u_{n}, v_{n}\right)\right\rangle+o_{n}(1) \\
& =\lambda_{n}(p-q)\left\|\left(u_{n}, v_{n}\right)\right\|_{\varepsilon}^{p}+o_{n}(1) .
\end{aligned}
$$

This expression and (2.3) imply that $\lambda_{n} \rightarrow 0$, and therefore $I_{\varepsilon}^{\prime}\left(u_{n}, v_{n}\right) \rightarrow 0$ in the dual space $X_{\varepsilon}^{*}$.

Since Palais-Smale sequences of $I_{\varepsilon}$ are bounded, up to a subsequence we have that $\left(u_{n}, v_{n}\right) \rightarrow(u, v)$ weakly in $X_{\varepsilon}$ with $(u, v)$ being a critical point of $I_{\varepsilon}$. Moreover, we can show that $I_{\varepsilon}^{\prime}\left(u_{n}-u, v_{n}-v\right) \rightarrow 0$ and

$$
\lim _{n \rightarrow \infty} I_{\varepsilon}\left(u_{n}-u, v_{n}-v\right)=d-I_{\varepsilon}(u, v)=\tilde{d}
$$

Recalling that $I_{\varepsilon}^{\prime}(u, v)=0$ we get

$$
I_{\varepsilon}(u, v)=I_{\varepsilon}(u, v)-\frac{1}{p}\left\langle I_{\varepsilon}^{\prime}(u, v),(u, v)\right\rangle=\frac{q-p}{p} \int Q(u, v) \geq 0
$$

and therefore $\tilde{d}<c_{\infty}$. If $\max \left\{V_{\infty}, W_{\infty}\right\}<\infty$, it follows from Lemma 2.4 that $\left(u_{n}-u, v_{n}-v\right) \rightarrow(0,0)$, i.e., $\left(u_{n}, v_{n}\right) \rightarrow(u, v)$ strongly in $X_{\varepsilon}$. In view of Corollary 2.5, the same occurs in the complementary case $\max \left\{V_{\infty}, W_{\infty}\right\}=\infty$. The proposition is proved.

Corollary 2.6. The critical points of the functional $I_{\varepsilon}$ constrained to $\mathcal{N}_{\varepsilon}$ are critical points of $I_{\varepsilon}$ in $X_{\varepsilon}$

Proof. It suffices to argue as in the second part of the above proof. We omit the details.

\section{Proof of Theorem 1.1.}

We commence with a technical result.

Lemma 3.1. Let $\varepsilon_{n} \rightarrow 0^{+}$and $\left(\left(u_{n}, v_{n}\right)\right) \subset \mathcal{N}_{\varepsilon_{n}}$ be such that $I_{\varepsilon_{n}}\left(u_{n}, v_{n}\right) \rightarrow c_{0}$. Then there exists $\left(\widetilde{y}_{n}\right) \subset \mathbb{R}^{N}$ such that the translated sequence

$$
\left(\widetilde{u}_{n}(x), \widetilde{v}_{n}(x)\right):=\left(u_{n}\left(x+\tilde{y}_{n}\right), v_{n}\left(x+\widetilde{y}_{n}\right)\right)
$$

has a subsequence which converges in $X_{0}$. Moreover, up to a subsequence, $\left(y_{n}\right):=$ $\left(\varepsilon_{n} \widetilde{y}_{n}\right)$ is such that $y_{n} \rightarrow y \in M$. 
Proof. Since $\left\langle I_{\varepsilon_{n}}^{\prime}\left(u_{n}, v_{n}\right),\left(u_{n}, v_{n}\right)\right\rangle=0$ and $I_{\varepsilon_{n}}\left(u_{n}, v_{n}\right) \rightarrow c_{0}$, we can proceed as in the proof of Proposition 2.2 to conclude that $\left(\left(u_{n}, v_{n}\right)\right)$ is bounded. Moreover, since $c_{0}>0$, we cannot have $\left\|\left(u_{n}, v_{n}\right)\right\|_{\varepsilon_{n}} \rightarrow 0$. Hence, arguing as in Lemma 2.1, we obtain a sequence $\left(\widetilde{y}_{n}\right) \subset \mathbb{R}^{N}$ such that

$$
\left(\widetilde{u}_{n}, \widetilde{v}_{n}\right) \rightarrow(\widetilde{u}, \widetilde{v}) \text { weakly in } X_{0},
$$

where $\left(\widetilde{u}_{n}(x), \widetilde{v}_{n}(x)\right):=\left(u_{n}\left(x+\widetilde{y}_{n}\right), v_{n}\left(x+\widetilde{y}_{n}\right)\right)$ and $(\widetilde{u}, \widetilde{v}) \neq(0,0)$.

Let $\left(t_{n}\right) \subset(0,+\infty)$ be such that $\left(\widehat{u}_{n}, \widehat{v}_{n}\right):=\left(t_{n} \widetilde{u}_{n}, t_{n} \widetilde{v}_{n}\right) \in \mathcal{N}_{0}$. If we set $y_{n}:=\varepsilon_{n} \widetilde{y}_{n}$ we can use the change of variables $z \mapsto x+\widetilde{y}_{n}$ to get

$$
\begin{aligned}
I_{0}\left(\widehat{u}_{n}, \widehat{v}_{n}\right) \leq & \frac{t_{n}^{p}}{p} \int\left(\left|\nabla \widetilde{u}_{n}\right|^{p}+\left|\nabla \widetilde{v}_{n}\right|^{p}\right)-\int Q\left(t_{n} \widetilde{u}_{n}, t_{n} \widetilde{v}_{n}\right) \\
& +\frac{t_{n}^{p}}{p} \int\left(V\left(\varepsilon_{n}\left(x+\widetilde{y}_{n}\right)\right)\left|\widetilde{u}_{n}\right|^{p}+W\left(\varepsilon_{n}\left(x+\widetilde{y}_{n}\right)\right)\left|\widetilde{v}_{n}\right|^{p}\right) \\
= & I_{\varepsilon_{n}}\left(t_{n} u_{n}, t_{n} u_{n}\right) \leq I_{\varepsilon_{n}}\left(u_{n}, v_{n}\right)=c_{0}+o_{n}(1) .
\end{aligned}
$$

Since $c_{0} \leq I_{0}\left(\widehat{u}_{n}, \widehat{v}_{n}\right)$ we conclude that $I_{0}\left(\widehat{u}_{n}, \widehat{v}_{n}\right) \rightarrow c_{0}$.

Since $\left(\left(\widetilde{u}_{n}, \widetilde{v}_{n}\right)\right)$ and $\left(\left(\widehat{u}_{n}, \widehat{v}_{n}\right)\right)$ are bounded and $\left(\widetilde{u}_{n}, \widetilde{v}_{n}\right) \nrightarrow(0,0)$, the sequence $\left(t_{n}\right)$ is bounded. Thus, up to a subsequence, $t_{n} \rightarrow t_{0} \geq 0$. If $t_{0}=0$ we can use the boundedness of $\left(\left(\widetilde{u}_{n}, \widetilde{v}_{n}\right)\right)$ to get $\left(\widehat{u}_{n}, \widehat{v}_{n}\right)=t_{n}\left(\widetilde{u}_{n}, \widetilde{v}_{n}\right) \rightarrow(0,0)$. Hence $I_{0}\left(\widehat{u}_{n}, \widehat{v}_{n}\right) \rightarrow 0$, which contradicts $c_{0}>0$. Thus, $t_{0}>0$. We notice that, up to a subsequence, $\left(\widehat{u}_{n}, \widehat{v}_{n}\right) \rightarrow t_{0}(\widetilde{u}, \widetilde{v})=(\widehat{u}, \widehat{v})$ weakly in $X_{0}$. Since $t_{0}>0$ and $(\widetilde{u}, \widetilde{v}) \neq(0,0)$, we have concluded that

$$
I_{0}\left(\widehat{u}_{n}, \widehat{v}_{n}\right) \rightarrow c_{0} \quad \text { and } \quad\left(\widehat{u}_{n}, \widehat{v}_{n}\right) \rightarrow(\widehat{u}, \widehat{v}) \neq(0,0) \text { weakly in } X_{0} .
$$

We can now use (2.1) and the same calculations performed in [1, Theorem 3.1] to conclude that $\left(\widehat{u}_{n}, \widehat{v}_{n}\right) \rightarrow(\widehat{u}, \widehat{v})$ in $X_{0}$, which implies that $\left(\widetilde{u}_{n}, \widetilde{v}_{n}\right) \rightarrow(\widetilde{u}, \widetilde{v})$ in $X_{0}$.

It remains to show that $\left(y_{n}\right)$ has a subsequence such that $y_{n} \rightarrow y \in M$. We start by proving that $\left(y_{n}\right)$ is bounded. Indeed, suppose by contradiction that there exists a subsequence, still denoted by $\left(y_{n}\right)$, such that $\left|y_{n}\right| \rightarrow+\infty$. We will obtain a contradiction by considering two cases.

Case $1 \max \left\{V_{\infty}, W_{\infty}\right\}=\infty$.

Since $\left(u_{n}, v_{n}\right) \in \mathcal{N}_{\varepsilon_{n}}$ we have that

$$
\begin{aligned}
q \int Q\left(u_{n}\left(x+\widetilde{y}_{n}\right), v_{n}\left(x+\widetilde{y}_{n}\right)\right) \geq & \int V\left(\varepsilon_{n} x+y_{n}\right)\left|u_{n}\left(x+\widetilde{y}_{n}\right)\right|^{p} \\
& +\int W\left(\varepsilon_{n} x+y_{n}\right)\left|v_{n}\left(x+\widetilde{y}_{n}\right)\right|^{p} .
\end{aligned}
$$

Applying Fatou's lemma we obtain

$$
\liminf _{n \rightarrow \infty} \int Q\left(u_{n}\left(x+\widetilde{y}_{n}\right), v_{n}\left(x+\widetilde{y}_{n}\right)\right) \geq \infty .
$$


On the other hand, the boundedness of $\left(\left(u_{n}, v_{n}\right)\right)$ and $(2.2)$, imply that the left hand side in the above expression is bounded. Thus, we obtain a contradiction.

Case $2 \max \left\{V_{\infty}, W_{\infty}\right\}<\infty$.

In this case, since $\left(\hat{u}_{n}, \hat{v}_{n}\right) \rightarrow(\hat{u}, \hat{v})$ strongly in $X_{0}$ and $V_{0}<\max \left\{V_{\infty}, W_{\infty}\right\}$ we have

$$
\begin{aligned}
c_{0}= & I_{0}(\widehat{u}, \widehat{v})<I_{\infty}(\widehat{u}, \widehat{v}) \\
\leq & \liminf _{n \rightarrow \infty}\left\{\frac{1}{p} \int\left(\left|\nabla \widehat{u}_{n}\right|^{p}+\left|\nabla \widehat{v}_{n}\right|^{p}\right)-\int Q\left(\widehat{u}_{n}, \widehat{v}_{n}\right)\right. \\
& \left.+\frac{1}{p} \int\left(V\left(\varepsilon_{n} x+y_{n}\right)\left|\widehat{u}_{n}\right|^{p}+W\left(\varepsilon_{n} x+y_{n}\right)\left|\widehat{v}_{n}\right|^{p}\right)\right\} \\
= & \liminf _{n \rightarrow \infty} I_{\varepsilon_{n}}\left(t_{n} u_{n}, t_{n} v_{n}\right) \leq \liminf _{n \rightarrow \infty} I_{\varepsilon_{n}}\left(u_{n}, v_{n}\right)=c_{0},
\end{aligned}
$$

which does not make sense.

We then conclude that $\left(y_{n}\right)$ is bounded and therefore, up to a subsequence, $y_{n} \rightarrow y$. If $y \notin M$ then $V_{0}<\max \{V(y), W(y)\}$ and we have that

$$
c_{0}<\frac{1}{p} \int\left(|\nabla \widehat{u}|^{p}+|\nabla \widehat{v}|^{p}+V(y)|\widehat{u}|^{p}+W(y)|\widehat{u}|^{p}\right)-\int Q(\widehat{u}, \widehat{v}) .
$$

This inequality and the same kind of calculations performed in (3.1) provide a contradiction. Thus, $y \in M$ and the lemma is proved.

Fix $\delta>0$ and choose a cut-off function $\eta \in C_{0}^{\infty}(\mathbb{R},[0,1])$ such that $\eta(s)=1$ if $0 \leq s \leq \delta / 2$ and $\eta(s)=0$ if $s \geq \delta$. Let $\left(\omega_{1}, \omega_{2}\right) \in X_{0}$ be the solution of $(A)$ given by Proposition 2.2. For each $y \in M$ we define

$$
\Psi_{i, \varepsilon, y}(x):=\eta(|\varepsilon x-y|) \omega_{i}\left(\frac{\varepsilon x-y}{\varepsilon}\right), \quad i=1,2 .
$$

If $t_{\varepsilon}$ denotes the unique positive number satisfying

$$
\max _{t \geq 0} I_{\varepsilon}\left(t \Psi_{1, \varepsilon, y}, t \Psi_{2, \varepsilon, y}\right)=I_{\varepsilon}\left(t_{\varepsilon} \Psi_{1, \varepsilon, y}, t_{\varepsilon} \Psi_{2, \varepsilon, y}\right),
$$

we introduce the map $\Phi_{\varepsilon}: M \rightarrow \mathcal{N}_{\varepsilon}$ by setting

$$
\Phi_{\varepsilon}(y):=\left(t_{\varepsilon} \Psi_{1, \varepsilon, y}, t_{\varepsilon} \Psi_{2, \varepsilon, y}\right) .
$$

Since $I_{0}\left(\omega_{1}, \omega_{2}\right)=c_{0}$ we can use the Lebesgue's theorem and the compactness of $M$ to check that

$$
\lim _{\varepsilon \rightarrow 0^{+}} I_{\varepsilon}\left(\Phi_{\varepsilon}(y)\right)=c_{0}, \quad \text { uniformly for } y \in M \text {. }
$$


We take now $\rho=\rho_{\delta}>0$ such that $M_{\delta} \subset B_{\rho}(0)$ and consider $\Upsilon: \mathbb{R}^{N} \rightarrow \mathbb{R}^{N}$ defined as $\Upsilon(x):=x$ for $|x|<\rho$ and $\Upsilon(x):=\rho x /|x|$ for $|x| \geq \rho$. We define the barycenter map $\beta_{\varepsilon}: \mathcal{N}_{\varepsilon} \rightarrow \mathbb{R}^{N}$ as being

$$
\beta_{\varepsilon}(u, v):=\frac{\int \Upsilon(\varepsilon x)\left(|u(x)|^{p}+|v(x)|^{p}\right) \mathrm{d} x}{\int\left(|u(x)|^{p}+|v(x)|^{p}\right) \mathrm{d} x} .
$$

Lemma 3.2. The function $\Phi_{\varepsilon}$ satisfies

$$
\lim _{\varepsilon \rightarrow 0^{+}} \beta_{\varepsilon}\left(\Phi_{\varepsilon}(y)\right)=y \quad \text { uniformly for } y \in M \text {. }
$$

Proof. Suppose, by contradiction, that the lemma is false. Then, there exist $\delta_{0}>0,\left(y_{n}\right) \subset M$ and $\varepsilon_{n} \rightarrow 0$ such that

$$
\left|\beta_{\varepsilon_{n}}\left(\Phi_{\varepsilon_{n}}\left(y_{n}\right)\right)-y_{n}\right| \geq \delta_{0} .
$$

By using the change of variables $z:=\left(\varepsilon_{n} x-y_{n}\right) / \varepsilon_{n}$, we can write

$$
\beta_{\varepsilon_{n}}\left(\Phi_{\varepsilon_{n}}\left(y_{n}\right)\right)=y_{n}+\frac{\int_{\mathbb{R}^{N}}\left(\Upsilon\left(\varepsilon_{n} z+y_{n}\right)-y_{n}\right)\left|\eta\left(\left|\varepsilon_{n} z\right|\right)\right|^{p}\left(\left|\omega_{1}(z)\right|^{p}+\left|\omega_{2}(z)\right|^{p}\right) \mathrm{d} z}{\int_{\mathbb{R}^{N}}\left|\eta\left(\left|\varepsilon_{n} z\right|\right)\right|^{p}\left(\left|\omega_{1}(z)\right|^{p}+\left|\omega_{2}(z)\right|^{p}\right) \mathrm{d} z} .
$$

Since $M \subset B_{\rho}(0)$ and $\left.\Upsilon\right|_{B_{\rho}(0)} \equiv \mathrm{Id}$, we can use the above expression and the Lebesgue's theorem to conclude that

$$
\left|\beta_{\varepsilon_{n}}\left(\Phi_{\varepsilon_{n}}\left(y_{n}\right)\right)-y_{n}\right|=o_{n}(1),
$$

which is a contradiction. The lemma is proved.

Following [11], we take a function $h:[0, \infty) \rightarrow[0, \infty)$ such that $h(\varepsilon) \rightarrow 0$ as $\varepsilon \rightarrow 0^{+}$and set

$$
\Sigma_{\varepsilon}:=\left\{(u, v) \in \mathcal{N}_{\varepsilon}: I_{\varepsilon}(u, v) \leq c_{0}+h(\varepsilon)\right\} .
$$

Given $y \in M$, we can use (3.2) to conclude that $h(\varepsilon)=\left|I_{\varepsilon}\left(\Phi_{\varepsilon}(y)\right)-c_{0}\right|$ is such that $h(\varepsilon) \rightarrow 0$ as $\varepsilon \rightarrow 0$. Thus, $\Phi_{\varepsilon}(y) \in \Sigma_{\varepsilon}$ and we have that $\Sigma_{\varepsilon} \neq \varnothing$ for any $\varepsilon>0$. Moreover, the following holds

Lemma 3.3. For any $\delta>0$ we have that

$$
\lim _{\varepsilon \rightarrow 0^{+}} \sup _{(u, v) \in \Sigma_{\varepsilon}} \operatorname{dist}\left(\beta_{\varepsilon}(u, v), M_{\delta}\right)=0 .
$$


Proof. Let $\left(\varepsilon_{n}\right) \subset \mathbb{R}$ be such that $\varepsilon_{n} \rightarrow 0^{+}$. By definition, there exists $\left(\left(u_{n}, v_{n}\right)\right) \subset$ $\Sigma_{\varepsilon_{n}}$ such that

$$
\operatorname{dist}\left(\beta_{\varepsilon_{n}}\left(u_{n}, v_{n}\right), M_{\delta}\right)=\sup _{(u, v) \in \Sigma_{\varepsilon_{n}}} \operatorname{dist}\left(\beta_{\varepsilon_{n}}(u, v), M_{\delta}\right)+o_{n}(1) .
$$

Thus, it suffices to find a sequence $\left(y_{n}\right) \subset M_{\delta}$ such that

$$
\left|\beta_{\varepsilon_{n}}\left(u_{n}, v_{n}\right)-y_{n}\right|=o_{n}(1) .
$$

Since $\left(\left(u_{n}, v_{n}\right)\right) \subset \Sigma_{\varepsilon_{n}} \subset \mathcal{N}_{\varepsilon_{n}}$, we have that

$$
c_{0} \leq c_{\varepsilon_{n}} \leq I_{\varepsilon_{n}}\left(u_{n}, v_{n}\right) \leq c_{0}+h\left(\varepsilon_{n}\right),
$$

and therefore $I_{\varepsilon_{n}}\left(u_{n}, v_{n}\right) \rightarrow c_{0}$. We may now invoke Lemma 3.1 to obtain a sequence $\left(\widetilde{y}_{n}\right) \subset \mathbb{R}^{N}$ such that $\left(y_{n}\right):=\left(\varepsilon_{n} \widetilde{y}_{n}\right) \subset M_{\delta}$. We set

$$
\left(\widetilde{u}_{n}(x), \widetilde{v}_{n}(x)\right):=\left(u_{n}\left(\varepsilon_{n} x+y_{n}\right), v_{n}\left(\varepsilon_{n} x+y_{n}\right)\right)
$$

and observe that, since $\left(\widetilde{u}_{n}, \widetilde{v}_{n}\right) \rightarrow(u, v)$ in $X_{0}$ and $\varepsilon_{n} x+y_{n} \rightarrow y \in M$, a direct calculation shows that $\beta_{\varepsilon_{n}}\left(u_{n}, v_{n}\right)=y_{n}+o_{n}(1)$. The lemma is proved.

We are now ready to present the proof of the multiplicity result in the subcritical case.

Proof of Theorem 1.1. Given $\delta>0$ we can use (3.2), (3.3), Lemma 3.3, and argue as in $[11$, Section 6$]$ to obtain $\varepsilon_{\delta}>0$ such that, for any $\varepsilon \in\left(0, \varepsilon_{\delta}\right)$, the diagram

$$
M \stackrel{\Phi_{\varepsilon}}{\longrightarrow} \Sigma_{\varepsilon} \stackrel{\beta_{\varepsilon}}{\longrightarrow} M_{\delta}
$$

is well defined and $\beta_{\varepsilon} \circ \Phi_{\varepsilon}$ is homotopically equivalent to the embedding $\iota: M \rightarrow$ $M_{\delta}$. Using the definition of $\Sigma_{\varepsilon}$ and taking $\varepsilon_{\delta}$ small if necessary, we may suppose that $I_{\varepsilon}$ satisfies the Palais-Smale condition in $\Sigma_{\varepsilon}$. Standard LjusternikSchnirelmann theory provides at least $\operatorname{cat}_{\Sigma_{\varepsilon}}\left(\Sigma_{\varepsilon}\right)$ critical points $\left(u_{i}, v_{i}\right)$ of $I_{\varepsilon}$ restricted to $\mathcal{N}_{\varepsilon}$. The same ideas contained in the proof of [8, Lemma 4.3] show that $\operatorname{cat}_{\Sigma_{\varepsilon}}\left(\Sigma_{\varepsilon}\right) \geq \operatorname{cat}_{M_{\delta}}(M)$. By using Corollary 2.6 and the arguments of the proof of Proposition 2.2 we conclude that $u_{i}>0, v_{i}>0$ and $\left(u_{i}, v_{i}\right)$ is a solution of $\left(\widehat{P}_{\varepsilon}\right)$. The theorem is proved.

\section{The critical case}

In this section we present the proof of Theorem 1.2. Since many calculations are adaptations to that presented in the two early sections, we will emphasize only the differences between the subcritical and the critical case. 
We first consider the critical version of the problem $(A)$, namely

$$
\left\{\begin{array}{l}
-\Delta_{p} u+V_{0}|u|^{p-2} u=Q_{u}(u, v)+\alpha|u|^{\alpha-2} u|v|^{\beta} \text { in } \mathbb{R}^{N}, \\
-\Delta_{p} v+W_{0}|v|^{p-2} v=Q_{v}(u, v)+\beta|u|^{\alpha}|v|^{\beta-2} v \text { in } \mathbb{R}^{N}, \\
u(x), v(x)>0 \text { for all } x \in \mathbb{R}^{N},
\end{array}\right.
$$

whose solutions are related with the critical points of $J_{0}: X_{0} \rightarrow \mathbb{R}$ defined as

$$
J_{0}(u, v):=\|(u, v)\|_{0}^{p}-\int Q(u, v)-\int\left(u^{+}\right)^{\alpha}\left(v^{+}\right)^{\beta} .
$$

We denote by $m_{0}$ the ground state level of $J_{0}$, that is,

$$
m_{0}:=\inf _{(u, v) \in X_{0} \backslash\{(0,0)\}} \max _{t \geq 0} J_{0}(t u, t v)>0 .
$$

As usual, we denote by $S$ the best constant of the embedding $W^{1, p}\left(\mathbb{R}^{N}\right) \hookrightarrow$ $L^{p^{*}}\left(\mathbb{R}^{N}\right)$, that is

$$
S:=\inf _{u \in W^{1, p}\left(\mathbb{R}^{N}\right) \backslash\{0\}} \frac{\int|\nabla u|^{p}}{\left(\int|u|^{p^{*}}\right)^{p / p^{*}}} .
$$

We also consider

$$
\widetilde{S}=\widetilde{S}(\alpha, \beta):=\inf _{u, v \in W^{1, p}\left(\mathbb{R}^{N}\right) \backslash\{0\}} \frac{\int|\nabla u|^{p}+\int|\nabla v|^{p}}{\left(\int|u|^{\alpha}|v|^{\beta}\right)^{p / p^{*}}} .
$$

The above number was introduced in [2], where the authors proved an interesting relation between $S$ and $\widetilde{S}$, namely

Lemma 4.1. The constants $S$ and $\widetilde{S}$ satisfies

$$
\widetilde{S}=\left[\left(\frac{\alpha}{\beta}\right)^{\beta / p^{*}}+\left(\frac{\beta}{\alpha}\right)^{\alpha / p^{*}}\right] S .
$$

Moreover, if $\omega$ realizes $S$, then $(A \omega, B \omega)$ realizes $\widetilde{S}$ for any constants $A$ and $B$ such that $(A / B)=(\alpha / \beta)^{1 / p}$.

Proof. The proof is similar to that presented in [2, Theorem 5].

Lemma 4.2. Let $\left(\left(u_{n}, v_{n}\right)\right) \subset X_{0}$ be a (PS $)_{d}$ sequence for the functional $J_{0}$ with $d<\frac{1}{N} \widetilde{S}^{N / p}$. Then we have either 
(i) $\left\|\left(u_{n}, v_{n}\right)\right\|_{0} \rightarrow 0$, or

(ii) there exists a sequence $\left(y_{n}\right) \subset \mathbb{R}^{N}$ and constants $R, \gamma>0$ such that

$$
\liminf _{n \rightarrow \infty} \int_{B_{R}\left(y_{n}\right)}\left(\left|u_{n}\right|^{p}+\left|v_{n}\right|^{p}\right) \geq \gamma>0 .
$$

Proof. Suppose that (ii) does not hold. Then, as in Lemma 2.1, we can prove that $\int Q\left(u_{n}, v_{n}\right) \rightarrow 0$. Since $\left(\left(u_{n}, v_{n}\right)\right)$ is bounded, $\left\langle J_{0}^{\prime}\left(u_{n}, v_{n}\right),\left(u_{n}, v_{n}\right)\right\rangle \rightarrow 0$. Taking a subsequence, we obtain $l \geq 0$ such that

$$
\left\|\left(u_{n}, v_{n}\right)\right\|_{0}^{p} \rightarrow l \text { and } p^{*} \int\left(u_{n}^{+}\right)^{\alpha}\left(v_{n}^{+}\right)^{\beta} \rightarrow l .
$$

Since $J_{0}\left(u_{n}, v_{n}\right) \rightarrow d$, we can use (4.1) to conclude that $l=N d$. Recalling the definition of $\widetilde{S}$ we get

$$
\left\|\left(u_{n}, v_{n}\right)\right\|_{0}^{p} \geq \widetilde{S}\left(\int\left|u_{n}\right|^{\alpha}\left|v_{n}\right|^{\beta}\right)^{p / p^{*}} \geq \widetilde{S}\left(\int\left(u_{n}^{+}\right)^{\alpha}\left(v_{n}^{+}\right)^{\beta}\right)^{p / p^{*}} .
$$

Taking the limit we conclude that $l \geq \widetilde{S} l^{p / p^{*}}$. If $l>0$ we obtain

$$
N d=l \geq \widetilde{S}^{N / p},
$$

which does not make sense. Hence $l=0$ and therefore (i) holds.

Proposition 4.3. The problem $(C A)$ has a weak solution.

Proof. Since $J_{0}$ has the Mountain Pass geometry, there exits $\left(\left(u_{n}, v_{n}\right)\right) \subset X_{0}$ such that

$$
J_{0}\left(u_{n}, v_{n}\right) \rightarrow m_{0} \text { and } J_{0}^{\prime}\left(u_{n}, v_{n}\right) \rightarrow 0 .
$$

We claim that the number $m_{0}$ satisfies

$$
m_{0}<\frac{1}{N} \widetilde{S}^{N / p}
$$

Assuming for a moment that this is true, we can use Lemma 4.2 and argue as in the proof of Proposition 2.2 to obtain the desired solution.

It remains to prove the claim. In view of the definition of $m_{0}$ it suffices to obtain $(u, v) \in X_{0}$ such that

$$
\max _{t \geq 0} J_{0}(t u, t v)<\frac{1}{N} \widetilde{S}^{N / p} .
$$

We proceed as in [16, Lemma 3] and firstly recall (see [29]) that, for any $\delta>0$, the instanton

$$
w_{\delta}(x):=\left[\delta N\left(\frac{N-p}{p-1}\right)^{p-1}\right]^{(N-p) / p^{2}}\left(\delta+|x|^{p /(p-1)}\right)^{(p-N) / p},
$$


satisfies the problem

$$
\left\{\begin{array}{l}
-\Delta_{p} w=w^{p^{*}-1} \text { in } \mathbb{R}^{N}, \\
w \in W^{1, p}\left(\mathbb{R}^{N}\right), \quad w(x)>0 \text { for all } x \in \mathbb{R}^{N},
\end{array}\right.
$$

and

$$
\int\left|\nabla w_{\delta}\right|^{p}=\int\left|w_{\delta}\right|^{p^{*}}=S^{N / p} .
$$

Let $A, B$ be positive constants such that $(A / B)=(\alpha / \beta)^{1 / p}$. By Lemma 4.1 we know that $\left(A w_{\delta}, B w_{\delta}\right)$ realizes $\widetilde{S}$. Hence, we can easily obtain the following relation between $S$ and $\widetilde{S}$

$$
\widetilde{S}=S \frac{\left(A^{p}+B^{p}\right)}{\left(A^{\alpha} B^{\beta}\right)^{p / p^{*}}} .
$$
Setting

Let $\eta \in C_{0}^{\infty}\left(\mathbb{R}^{N},[0,1]\right)$ be such that $\eta \equiv 1$ on $B_{1}(0)$ and $\eta \equiv 0$ on $\mathbb{R}^{N} \backslash B_{2}(0)$.

$$
\psi_{\delta}(x):=\frac{\eta(x) w_{\delta}(x)}{\left|\eta w_{\delta}\right|_{p^{*}}},
$$

we can use the definition of $\psi_{\delta}$ and $\left(Q_{5}\right)$ to get

$$
J_{0}\left(t A \psi_{\delta}, t B \psi_{\delta}\right) \leq \frac{t^{p}}{p} D_{\delta}\left(A^{p}+B^{p}\right)-\frac{t^{p^{*}}}{p^{*}} A^{\alpha} B^{\beta}-\lambda t^{q_{1}} A^{q_{1}} B^{q_{1}} \int_{B_{2}(0)}\left|\psi_{\delta}\right|^{q_{1}},
$$

where $q_{1} \in\left(p, p^{*}\right)$ is given by condition $\left(Q_{5}\right)$ and

$$
D_{\delta}=\int\left|\nabla \psi_{\delta}\right|^{p}+V_{0}\left|\psi_{\delta}\right|^{p} .
$$

Let $h_{\delta}(t)$ be the $t$-function on the right hand side of the above expression and denote by $t_{\delta}$ the maximum point of $h_{\delta}$ on $(0, \infty)$. Since $h_{\delta}^{\prime}\left(t_{\delta}\right)=0$ we have that

$$
\bar{t}_{\delta}:=\left[\frac{D_{\delta}\left(A^{p}+B^{p}\right)}{A^{\alpha} B^{\beta}}\right]^{1 /\left(p^{*}-p\right)} \geq t_{\delta}>0 .
$$

Since the function $t \mapsto t^{p} D_{\delta}\left(A^{p}+B^{p}\right) / p-t^{p^{*}} A^{\alpha} B^{\beta} / p^{*}$ is increasing in $\left(0, \bar{t}_{\delta}\right)$, we can use the definition of $h_{\delta}$ to get

$$
h_{\delta}\left(t_{\delta}\right) \leq \frac{1}{N}\left[\frac{D_{\delta}\left(A^{p}+B^{p}\right)}{\left(A^{\alpha} B^{\beta}\right)^{p / p^{*}}}\right]^{N / p}-\lambda t^{q_{1}} A^{q_{1}} B^{q_{1}} \int_{B_{2}(0)}\left|\psi_{\delta}\right|^{q_{1}} .
$$

If $a, b \geq 0$ and $s \geq 1$, then $(a+b)^{s} \leq a^{s}+s(a+b)^{s-1} b$. Therefore, there exists $C_{1}>0$ such that

$$
D_{\delta}^{N / p} \leq S^{N / p}+O\left(\delta^{(N-p) / p}\right)+C_{1} \int_{B_{2}(0)}\left|\psi_{\delta}\right|^{p} .
$$


Moreover, we can obtain $\rho>0$ such that $t_{\delta}>\rho$ for any $\delta$ small. Hence, it follows from the above inequality, (4.3) and (4.2) that

$$
h_{\delta}\left(t_{\delta}\right) \leq \frac{1}{N} \widetilde{S}^{N / p}+\delta^{(N-p) / p}\left[C_{2}+\frac{C_{3}}{\delta^{(N-p) / p}}\left(\int_{B_{2}(0)}\left|\psi_{\delta}\right|^{p}-\lambda C_{4}\left|\psi_{\delta}\right|^{q_{1}}\right)\right],
$$

for positive constants $C_{2}, C_{3}$ and $C_{4}$. In view of the hypotheses on $\lambda>0$ given in $\left(Q_{5}\right)$, we can argue as in the proof of [25, Claim 2] to check that, if $\delta$ is sufficiently small, the second term in the right hand side above is negative. Thus,

$$
\max _{t \geq 0} J_{0}\left(t A \psi_{\delta}, t B \psi_{\delta}\right) \leq \max _{t \geq 0} h_{\delta}(t)=h_{\delta}\left(t_{\delta}\right)<\frac{1}{N} \widetilde{S}^{N / p}
$$

and the proposition is proved.

In order to obtain solutions for $\left(C P_{\varepsilon}\right)$ we will consider the system

$$
\left\{\begin{array}{l}
-\Delta_{p} u+V(\varepsilon x)|u|^{p-2} u=Q_{u}(u, v)+\alpha|u|^{\alpha-2} u|v|^{\beta} \text { in } \mathbb{R}^{N}, \\
-\Delta_{p} v+W(\varepsilon x)|v|^{p-2} v=Q_{v}(u, v)+\beta|u|^{\alpha}|v|^{\beta-2} v \text { in } \mathbb{R}^{N} \\
u(x), v(x)>0 \text { for all } x \in \mathbb{R}^{N}
\end{array}\right.
$$

and to look for critical points of the functional $J_{\varepsilon}: X_{\varepsilon} \rightarrow \mathbb{R}$ given by

$$
J_{\varepsilon}(u, v):=\frac{1}{p}\|(u, v)\|_{\varepsilon}^{p}-\int Q(u, v)-\int\left(u^{+}\right)^{\alpha}\left(v^{+}\right)^{\beta},
$$

where $X_{\varepsilon}$ is the same space defined at the beginning of Section 2 .

The critical points of $J_{\varepsilon}$ belong to the Nehari manifold

$$
\mathcal{M}_{\varepsilon}:=\left\{(u, v) \in X_{\varepsilon} \backslash\{(0,0)\}:\left\langle J_{\varepsilon}^{\prime}(u, v),(u, v)\right\rangle=0\right\}
$$

and the ground state level is given by

$$
m_{\varepsilon}:=\inf _{(u, v) \in \mathcal{M}_{\varepsilon}} J_{\varepsilon}(u, v)=\inf _{(u, v) \in X_{\varepsilon} \backslash\{(0,0)\}} \max _{t \geq 0} J_{\varepsilon}(t u, t v)>0 .
$$

As before, the Palais-Smale condition for the functional $J_{\varepsilon}$ is related with $V_{\infty}$ and $W_{\infty}$. When these two quantities are finite we define the limit functional $J_{\infty}: X_{0} \rightarrow \mathbb{R}$ as being

$J_{\infty}(u, v):=\frac{1}{p} \int\left(|\nabla u|^{p}+|\nabla v|^{p}+V_{\infty}|u|^{p}+W_{\infty}|v|^{p}\right)-\int Q(u, v)-\int\left(u^{+}\right)^{\alpha}\left(v^{+}\right)^{\beta}$,

and its ground state level

$$
m_{\infty}:=\inf _{(u, v) \in X_{0} \backslash\{(0,0)\}} \max _{t \geq 0} J_{\infty}(t u, t v)>0 .
$$


If $\max \left\{V_{\infty}, W_{\infty}\right\}=\infty$, we set $m_{\infty}:=\infty$.

Since the function $(u, v) \mapsto \int\left(u^{+}\right)^{\alpha}\left(v^{+}\right)^{\beta}$ is positively $p^{*}$-homogeneous, we can argue as in Subsection 2.2 to get a compactness result for the functional $J_{\varepsilon}$. We only notice that, in this new setting, we need to use Lemma 4.2 instead of Lemma 2.1. Hence, the following result holds.

Proposition 4.4. The functional $J_{\varepsilon}$ constrained to $\mathcal{M}_{\varepsilon}$ satisfies the $(\mathrm{PS})_{d}$ condition at any level $d<\min \left\{m_{\infty}, \widetilde{S}^{N / p} / N\right\}$. Moreover, critical points of $J_{\varepsilon}$ constrained to $\mathcal{M}_{\varepsilon}$ are critical points of $J_{\varepsilon}$ in $X_{\varepsilon}$.

We are now ready to prove our second multiplicity result.

Proof of Theorem 1.2. Since the proof is very similar to that presented for Theorem 1.1, we only sketch it. Fix $\delta>0$ and choose $\eta \in C_{0}^{\infty}(\mathbb{R},[0,1])$ such that $\eta(s)=1$ if $0 \leq s \leq \delta / 2$ and $\eta(s)=0$ if $s \geq \delta$. Let $\left(\widetilde{\omega}_{1}, \widetilde{\omega}_{2}\right) \in X_{0}$ be the solution of $(C A)$ given by Proposition 4.3 and define, for each $y \in M$,

$$
\widetilde{\Psi}_{i, \varepsilon, y}(x):=\eta(|\varepsilon x-y|) \widetilde{\omega}_{i}\left(\frac{\varepsilon x-y}{\varepsilon}\right), \quad i=1,2 .
$$

We introduce the map $\widetilde{\Phi}_{\varepsilon}: M \rightarrow \mathcal{M}_{\varepsilon}$ by setting

$$
\widetilde{\Phi}_{\varepsilon}(y):=\left(\widetilde{t}_{\varepsilon} \widetilde{\Psi}_{1, \varepsilon, y}, \widetilde{t}_{\varepsilon} \widetilde{\Psi}_{2, \varepsilon, y}\right),
$$

where $\widetilde{t}_{\varepsilon}$ is the unique positive number satisfying

$$
\max _{t \geq 0} J_{\varepsilon}\left(t \widetilde{\Psi}_{1, \varepsilon, y}, t \widetilde{\Psi}_{2, \varepsilon, y}\right)=J_{\varepsilon}\left(\widetilde{t}_{\varepsilon} \widetilde{\Psi}_{1, \varepsilon, y}, \widetilde{t}_{\varepsilon} \widetilde{\Psi}_{2, \varepsilon, y}\right) .
$$

The following holds

$$
\lim _{\varepsilon \rightarrow 0^{+}} J_{\varepsilon}\left(\widetilde{\Phi}_{\varepsilon}(y)\right)=m_{0} \text { uniformly for } y \in M .
$$

Let $\Upsilon: \mathbb{R}^{N} \rightarrow \mathbb{R}^{N}$ be the function defined in Section 3 and consider the barycenter map $\widetilde{\beta}_{\varepsilon}: \mathcal{M}_{\varepsilon} \rightarrow \mathbb{R}^{N}$ given by

$$
\widetilde{\beta}_{\varepsilon}(u, v):=\frac{\int \Upsilon(\varepsilon x)\left(|u(x)|^{p}+|v(x)|^{p}\right) \mathrm{d} x}{\int\left(|u(x)|^{p}+|v(x)|^{p}\right) \mathrm{d} x} .
$$

As before we can check that

$$
\lim _{\varepsilon \rightarrow 0^{+}} \widetilde{\beta}_{\varepsilon}\left(\Phi_{\varepsilon}(y)\right)=y \text { uniformly for } y \in M
$$

and

$$
\lim _{\varepsilon \rightarrow 0^{+}} \sup _{(u, v) \in \widetilde{\Sigma}_{\varepsilon}} \operatorname{dist}\left(\widetilde{\beta}_{\varepsilon}(u, v), M_{\delta}\right)=0,
$$


where

$$
\widetilde{\Sigma}_{\varepsilon}:=\left\{(u, v) \in \mathcal{M}_{\varepsilon}: J_{\varepsilon}(u, v) \leq m_{0}+\widetilde{h}(\varepsilon)\right\}
$$

and $h:[0, \infty) \rightarrow[0, \infty)$ satisfies $h(\varepsilon) \rightarrow 0$ as $\varepsilon \rightarrow 0^{+}$.

The above equations provide $\varepsilon_{\delta}>0$ such that, for any $\varepsilon \in\left(0, \varepsilon_{\delta}\right)$, the diagram

$$
M \stackrel{\widetilde{\Phi}_{\varepsilon}}{\longrightarrow} \widetilde{\Sigma}_{\varepsilon} \stackrel{\widetilde{\beta}_{\varepsilon}}{\longrightarrow} M_{\delta}
$$

is well defined and $\widetilde{\beta}_{\varepsilon} \circ \widetilde{\Phi}_{\varepsilon}$ is homotopically equivalent to the embedding $\iota: M \rightarrow$ $M_{\delta}$. Hence we conclude that $\operatorname{cat}_{\widetilde{\Sigma}_{\varepsilon}}\left(\Sigma_{\varepsilon}\right) \geq \operatorname{cat}_{M_{\delta}}(M)$. In view of Proposition 4.4 and recalling that

$$
m_{0}<\frac{1}{N} \widetilde{S}^{N / p},
$$

we may suppose that $\varepsilon_{\delta}$ is small in such way that $J_{\varepsilon}$ satisfies the Palais-Smale condition in $\widetilde{\Sigma}_{\varepsilon}$. The proof now follows from Ljusternik-Schnirelmann theory and the same arguments used in the subcritical case.

\section{Acknowledgment}

The authors are grateful to the referee for his/her useful suggestions.

\section{References}

[1] C. O. Alves, Existence and multiplicity of solutions for a class of quasilinear equations, Adv. Non. Studies 5 (2005), 73-87.

[2] C. O. Alves, D. C. De Morais Filho and M. A. S. Souto, On systems of elliptic equations involving subcritical and critical Sobolev exponents, Nonlinear Analysis 42 (2000), 771-787.

[3] C. O. Alves and S. H. M. Soares, Existence and concentration of positive solutions for a class of gradient systems, NoDEA Nonlinear Differential Equations and Appl. 12 (2005), 437-457.

[4] A. Ambrosetti, M. Badiale and S. Cingolani, Semiclassical states of nonlinear Schrödinger equations, Arch. Rational Mech. Anal. 140 (1997), 285-300.

[5] A. Ávila and J. Yang, Multiple solutions of nonlinear elliptic systems, NoDEA Nonlinear Differential Equations and Appl. 12 (2005), 459-479.

[6] T. Bartsch and Z. Q. Wang, Multiple positive solutions for a nonlinear Schrödinger equation, Z. angew. Math. Phys. 51 (2000), 366-384.

[7] V. Benci and G. Cerami, The effect of the domain topology on the number of positive solutions of nonlinear elliptic problems, Arch. Rational Mech. Anal. 114 (1991), 79-93.

[8] V. Benci and G. Cerami, Multiple positive solutions of some elliptic problems via the Morse theory and the domain topology, Cal. Var. Partial Differential Equations 2 (1994), 29-48. 
[9] L. Boccardo and D. G. De Figueiredo, Some remarks on a system of quasilinear elliptic equations, NoDEA Nonlinear Differential Equations and Appl. 9 (2002), 309-323.

[10] H. Brezis and L. Nirenberg, Positive solutions of nonlinear elliptic equations involving critical Sobolev exponents, Comm. Pure Appl. Math. 36 (1983), 437-477.

[11] S. Cingolani and M. Lazzo, Multiple semiclassical standing waves for a class of nonlinear Schrödinger equations, Topol. Methods Nonlinear Anal. 10 (1997), 1-13.

[12] M. Clapp and Y. Ding, Positive solutions of a Schrödinger equation with critical nonlinearity, Z. angew. Math. Phys. 55 (2004), 592-605.

[13] M. Del Pino and P. Felmer, Local Mountain Pass for semilinear elliptic problems in unbounded domains, Calc. Var. Partial Differential Equations 4 (1996), 121-137.

[14] M. Del Pino and P. Felmer, Semi-classical states for nonlinear Schrödinger equations, J. Funct. Anal. 149 (1997), 245-265.

[15] M. Del Pino and P. Felmer, Semi-classical states of nonlinear Schrödinger equations: a variational reduction method, Math. Ann. 324 (2002), 1-32.

[16] D. C. De Morais Filho, J. M. Do Ó and M. A. S. Souto, A compactness embedding lemma, a principle of symmetric criticality and applications to elliptic problems, Proyecciones 19 (2000), 1-17.

[17] D. C. De Morais Filho and M. A. S. Souto, Systems of p-Laplacian equations involving homogeneous nonlinearities with critical Sobolev exponent degrees, Commun. in Partial Differential Equations 24 (1999), 1537-1553.

[18] G. M. Figueiredo, Multiplicity of solutions for a class of elliptic systems in $\mathbb{R}^{N}$, Electron. J. Differential Equations 76 (2006), 1-12.

[19] A. Floer and A. Weinstein, Nonspreading wave packets for the cubic Schrödinger equation with a bounded potential, J. Funct. Anal. 69 (1986), 397-408.

[20] G. B. Li, Some properties of weak solutions of nonlinear scalar fields equations, Ann. Acad. Sci. Fenn. Ser. A I Math. 15 (1990), 27-36.

[21] T. Lin and J. Wei, Spikes in two coupled nonlinear Schrödinger equations, Ann. Inst. H. PoincaréAnal. Non Linaire 22 (2005), 403-439.

[22] T. Lin and J. Wei, Spikes in two-component systems of nonlinear Schrödinger equations with trapping potentials, J. Differential Equations 229 (2006), $538-569$.

[23] T. Lin and J. Wei, Symbiotic bright solitary wave solutions of coupled nonlinear Schrödinger equations, Nonlinearity 19 (2006), 2755-2773.

[24] P. L. Lions, The concentration-compacteness principle in the calculus of variation. The locally compact case. II , Ann. Inst. H. Poincare Anal. Non Linéaire 1 (1984), 223-283.

[25] O. H. Miyagaki, On a class of semilinear elliptic problems in $\mathbb{R}^{N}$ with critical growth, Nonlinear Anal. 29 (1997), 773-781. 
[26] Y. G. Oh, Existence of semiclassical bound states of nonlinear Schrödinger equations with potentials of the class $(V)_{a}$, Comm. Partial Differential Equations 13 (1988), 1499-1519.

[27] Y. G. Oh, Correction to: Existence of semiclassical bound states of nonlinear Schrödinger equations with potentials of the class $(V)_{a}$, Comm. Partial Differential Equations 14 (1989), 833-834.

[28] P. H. Rabinowitz, On a class of nonlinear Schrödinger equations, Z. Angew Math. Phys. 43 (1992), 270-291.

[29] G. Talenti, Best constant in Sobolev inequality, Ann. Mat. Pura Appl. 110 (1976), 353-372.

[30] N. S. Trudinger, On Harnack type inequalities and their application to quasilinear elliptic equations, Comm. Pure Appl. Math. 20 (1967), 721-747.

[31] Y. Wan and A. Ávila, Multiple solutions of a coupled nonlinear Schrödinger system, J. Math. Anal. Appl. in press.

[32] X. Wang, On concentration of positive bound states of nonlinear Schrödinger equations, Comm. Math. Phys. 153 (1993), 229-244.

[33] X. Wang and B. Zeng, On concentration of positive bound states of nonlinear Schrödinger equations with competing potential functions, SIAM J. Math. Anal. 28 (1997), 633-655.

[34] M. Willem, Minimax theorems, Birkhäuser Boston, 1996.

[35] J. Yang, Positive solutions of quasilinear elliptic obstacle problems with critical exponents, Nonlinear Anal. 25 (1995), 1283-1306.

Giovany M. Figueiredo

Universidade Federal do Pará

Departamento de Matemática

66075-100, Belém-PA, Brazil

e-mail: giovany@ufpa.br

Marcelo F. Furtado

Universidade de Brasília

Departamento de Matemática

70910-900, Brasília-DF, Brazil

e-mail: mfurtado@unb.br

Received: 23 April 2007

Revised: 18 August 2007

Accepted: 23 October 2007

Published Online First 03 October 2008 
